\title{
Meilensteine der personalisierten Medizin bei Pemphigus und Pemphigoid
}

\author{
Katja Bieber ${ }^{\mathrm{a}} \quad$ Khalaf Kridin $^{\mathrm{a}}$ Shirin Emtenani ${ }^{\mathrm{a}}$ Katharina Boch $^{\mathrm{b}}$ Enno Schmidt ${ }^{\mathrm{a}} \mathrm{b}$ \\ Ralf J. Ludwiga, b
}

aübecker Institut für Experimentelle Dermatologie und Center for Research on Inflammation of the Skin, Universität Lübeck, Lübeck, Deutschland; ${ }^{\mathrm{b}}$ Abteilung für Dermatologie und Center for Research on Inflammation of the Skin, Universität Lübeck, Lübeck, Deutschland

\author{
Schlüsselwörter \\ Diagnose · Pemphigoid · Pemphigus · Präzisionsmedizin . \\ Therapie
}

\section{Zusammenfassung}

Pemphigus und Pemphigoid sind bullöse Autoimmunerkrankungen, deren Kennzeichen und Ursache Autoantikörper gegen Adhäsionsmoleküle in der Haut und/oder den Schleimhäuten sind. Das neue medizinische Modell der personalisierten Medizin, bei dem die Patienten in unterschiedliche Gruppen eingeteilt werden, zielt darauf ab, medizinische Entscheidungen, Vorgehensweisen und Interventionen an der voraussichtlichen Reaktion oder den Risikofaktoren des einzelnen Patienten auszurichten. Wichtige Meilensteine in der personalisierten Medizin bei Pemphigus und Pemphigoid waren der Nachweis der diesen Krankheiten zugrunde liegenden Autoimmunpathogenese sowie die Identifizierung und Klonung verschiedener Pemphigus/Pemphigoid-Autoantigene. Letztere bildet die Grundlage der aktuellen molekularen Diagnostik, die die Differenzierung von etwa einem Dutzend Pemphigus- und Pemphigoid-Entitäten ermöglicht. Die Bedeutung der Autoantigen-Identifizierung bei Pemphigus/Pemphigoid wird durch die Entwicklung autoantigenspezifischer Strategien zur BZell-Depletion zusätzlich unterstrichen. Um dieses Ziel zu errei- chen, wurde die Technologie der chimären Antigenrezeptor (CAR)-T-Zellen, die bei der Behandlung von bestimmten malignen hämatologischen Erkrankungen zur Anwendung kommt, adaptiert, indem chimäre Autoantigenrezeptor (CAAR)-T-Zellen generiert wurden. Neben diesen eher grundlagenwissenschaftlichen Meilensteinen der personalisierten Medizin bei Pemphigus und Pemphigoid spielen bei der personalisierten Medizin auch eine sorgfältige klinische Beobachtung sowie die Epidemiologie eine Rolle. Für Letzteres ist die Identifizierung klar abgegrenzter klinischer Phänotypen bei Pemphigoid, wie das nicht-entzündliche und das Gliptin-assoziierte bullöse Pemphigoid, ein wichtiges Beispiel. Die vorliegende Arbeit gibt einen Überblick über die spannenden Entwicklungen in der Grundlagenforschung sowie in der translationalen, klinischen und epidemiologischen Forschung zu Pemphigus und Pemphigoid. Auf diese Weise möchten wir mehr Forscher und Kliniker für dieses faszinierende und dynamische Forschungsgebiet begeistern.

(C) 20202021 Bieber, Kridin, Emtenani, Boch, Schmidt und Ludwig

\section{Pemphigus und Pemphigoid}

Pemphigus und Pemphigoid sind Autoimmunerkrankungen der Haut und/oder Schleimhäute, deren Kennzeichen und Ursache Autoantikörper gegen Strukturproteine sind [1]. Die Diagnose der beim jeweiligen Patienten vorliegenden spezifischen Pemphi- gus- oder Pemphigoid-Erkrankung erfolgt auf Grundlage des klinischen Bildes sowie durch den Nachweis gewebegebundener Autoantikörper und der Autoantikörperspezifität [2-4]. Pemphigus- und Pemphigoid-Erkrankungen sind zwar selten, doch gehen sie mit einer starken Krankheitsbelastung und einem hohen ungedeckten medizinischen Bedarf einher [5, 6]. Klinisches 


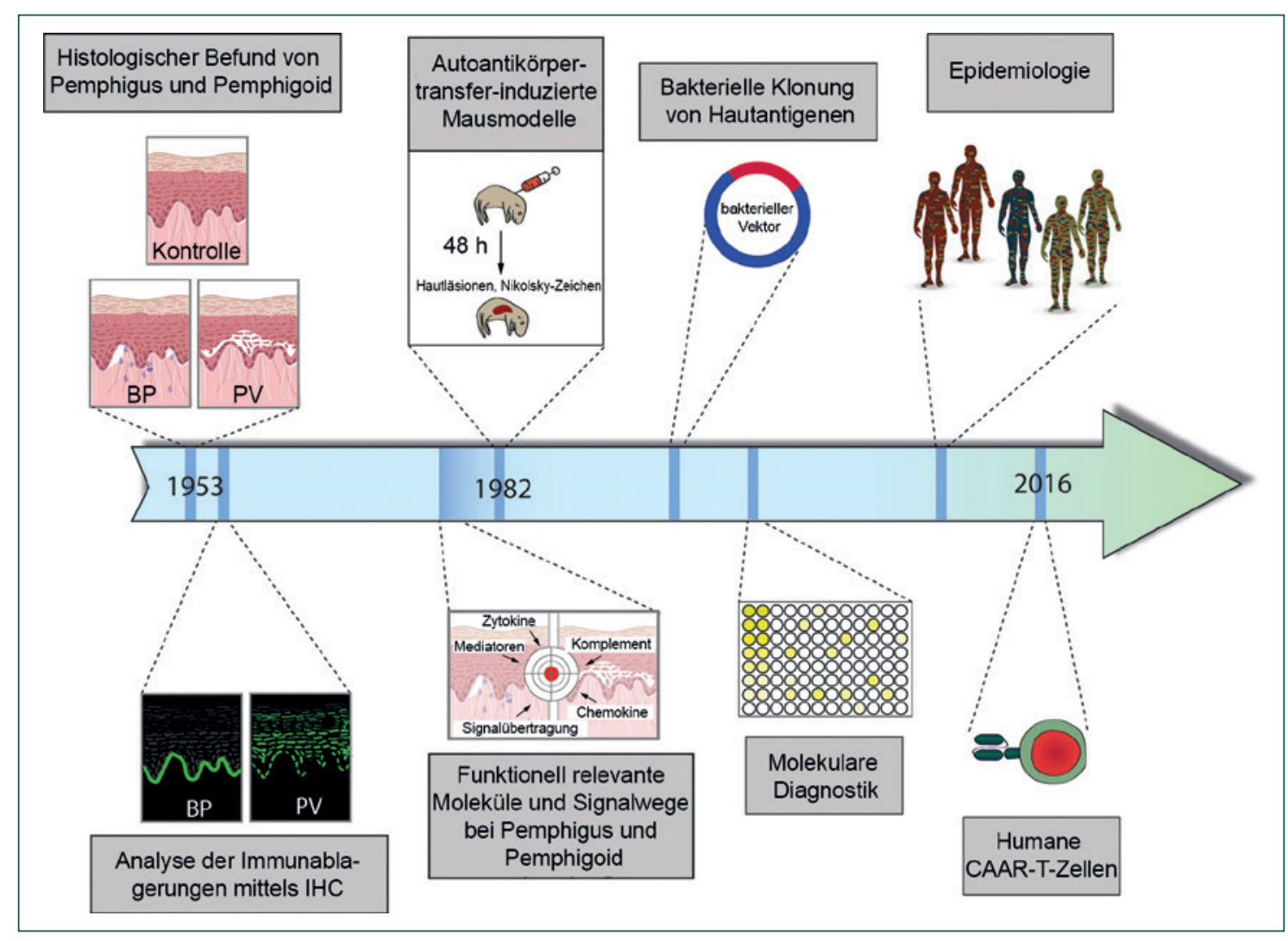

Abb. 1. Schematische Darstellung der Meilensteine der personalisierten Medizin bei Pemphigus und Pemphigoid. (a) Unterscheidung von Pemphigus und Pemphigoid anhand der klinischen Befunde und der Histologie der Hautläsionen. (b) Identifizierung unterschiedlicher Bindungsmuster von Autoantikörpern bei Pemphigus (beispielhaft: Pemphigus vulgaris, PV) und Pemphigoid (beispielhaft: bullöses Pemphigoid, BP). (c) Nachweis der Autoimmunpathogenese von Pemphigus und Pemphigoid durch Transfer von Patienten-IgG auf Mäuse, die anschließend Pemphigus entwickelten. (d) Identifizierung und Klonung von Autoantigenen. (e) Etablierung der derzeitigen molekularen Diagnostik bei Pemphigus und Pemphigoid. (f) Identifizierung eindeutiger Untergruppen durch sorgfältige klinische Beobachtung und Epidemiologie. (g) Individualisierte Behandlung durch Anwendung von chimären Autoantigenrezeptor (CAAR)-T-Zellen. Weitere Einzelheiten siehe Text.

Kennzeichen von Pemphigus und Pemphigoid ist die (muko)-kutane Blasenbildung, die bei Pemphigus intradermal und bei Pemphigoid subepidermal auftritt. Bei beiden Krankheiten kommt es zu einer CD4-abhängigen Autoantikörperbildung. Prinzipiell entstehen die Blasen bei Pemphigus als direkte Folge der Autoantikörperbindung an die Zielantigene und über komplement unabhängige Mechanismen, wohingegen die Blasenbildung bei Pemphigoid normalerweise von der Aktivierung angeborener Immunantworten durch den Fc-Teil der Autoantikörper abhängig ist [7, 8]. Pemphigus wird derzeit mit Kortikosteroiden in hohen Dosierungen und dem Anti-CD20-Antikörper Rituximab behandelt, wodurch $80 \%$ der Patienten eine vollständige Remission erreichen [9]. Die wirksamste Therapie des bullösen Pemphigoids (BP), der bei weitem häufigsten Pemphigoid-Erkrankung, besteht in der Langzeitbehandlung mit hochpotenten topischen oder oralen Kortikosteroiden [10]. Eine weitere durch einen chronischen Verlauf gekennzeichnete Pemphigoid-Erkrankung ist die im Vergleich zu BP oft stärker therapierefraktäre Epidermolysis bullosa acquisita (EBA). Hauptschwierigkeiten bei der Behandlung von Pemphigus sind die relativ lange Zeit, die es dauert, eine Remis- sion zu erzielen, die hohe Nebenwirkungsrate und Rezidive nach Absetzen der Behandlung [11]. Bei BP stellen Rezidive nach Absetzen der Behandlung die größte Herausforderung dar, denn diese haben eine längerfristige Kortikosteroidtherapie zur Folge, die zum Teil für die erhöhte Morbidität und Mortalität der Patienten verantwortlich ist [12].

Grundlage der aktuellen Forschung zu Pemphigus- und Pemphigoid-Erkrankungen ist unserer Ansicht nach die wegweisende Entdeckung von Walter Lever im Jahr 1953. Dieser nahm erstmals eine klare Unterscheidung zwischen Pemphigus und Pemphigoid-Erkrankungen vor allem auf Grundlage des histologischen Befundes der von der jeweiligen Krankheit befallenen Haut vor [13]. Diese Unterscheidung zwischen Pemphigus und Pemphigoid entsprechend der Histopathologie der Läsionen förderte die Entwicklung maßgeschneiderter Behandlungen für Patienten mit bullösen Autoimmunerkrankungen (autoimmune bullous diseases, AIBD), denn Patienten mit Pemphigus benötigen eine aggressivere immunsuppressive Therapie als Patienten mit BP. In der Folgezeit wurden weitere Meilensteine in der personalisierten Medizin bei Pemphigus und Pemphigoid gelegt (Abbildung 1): 

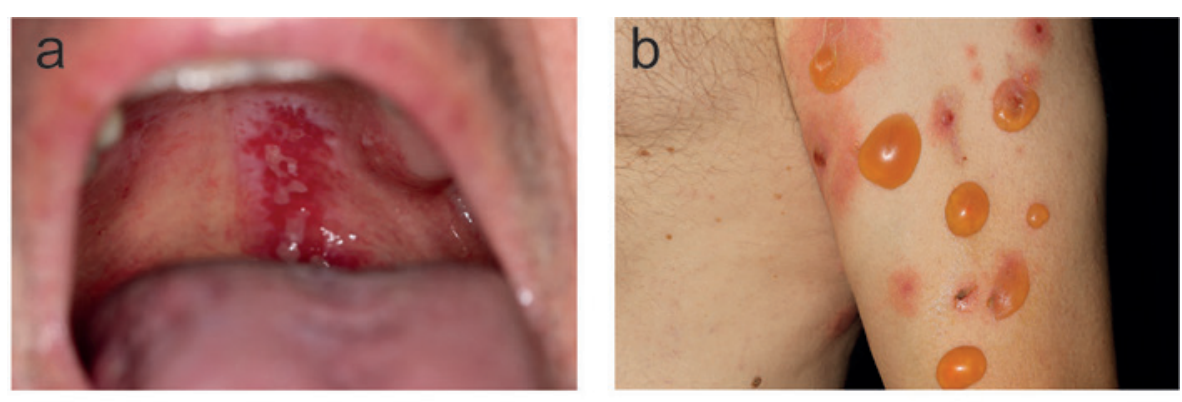

Abb. 2. Klinische, histologische und immunologische Merkmale von Pemphigus und Pemphigoid. (a) Klinisches Bild eines Pemphigus vulgaris mit Erosionen am oberen Gaumen. (b) Klinisches Bild eines bullösen Pemphigoids mit Blasenbildung auf erythematöser oder ansonsten scheinbar gesunder Haut. (c) Histopathologie einer Läsion eines Patienten mit Pemphigus vulgaris, die eine suprabasale Spaltbildung mit einer gewissen Akantholyse und die typische «Reihe von Grabsteinen» zeigt (Pfeile). (d) Biopsie einer Läsion von einem Patienten mit bullösem Pemphigoid; es ist eine subepidermale Spaltbildung erkennbar. Interessanterweise liegt in diesem Fall nur ein geringes dermales Infiltrat vor. (e) Gewebegebundenes IgG in einer periläsionalen Hautbiopsie eines Patienten mit Pemphigus vulgaris, die lgG-Ablagerungen mit einem honigwabenartigen Muster innerhalb der Epidermis zeigt. (f) Gewebegebundenes IgG in einer periläsionalen Hautbiopsie eines Patienten mit bullösem Pemphigoid, die lineare lgGAblagerungen entlang der dermoepidermalen Junktionszone zeigt.
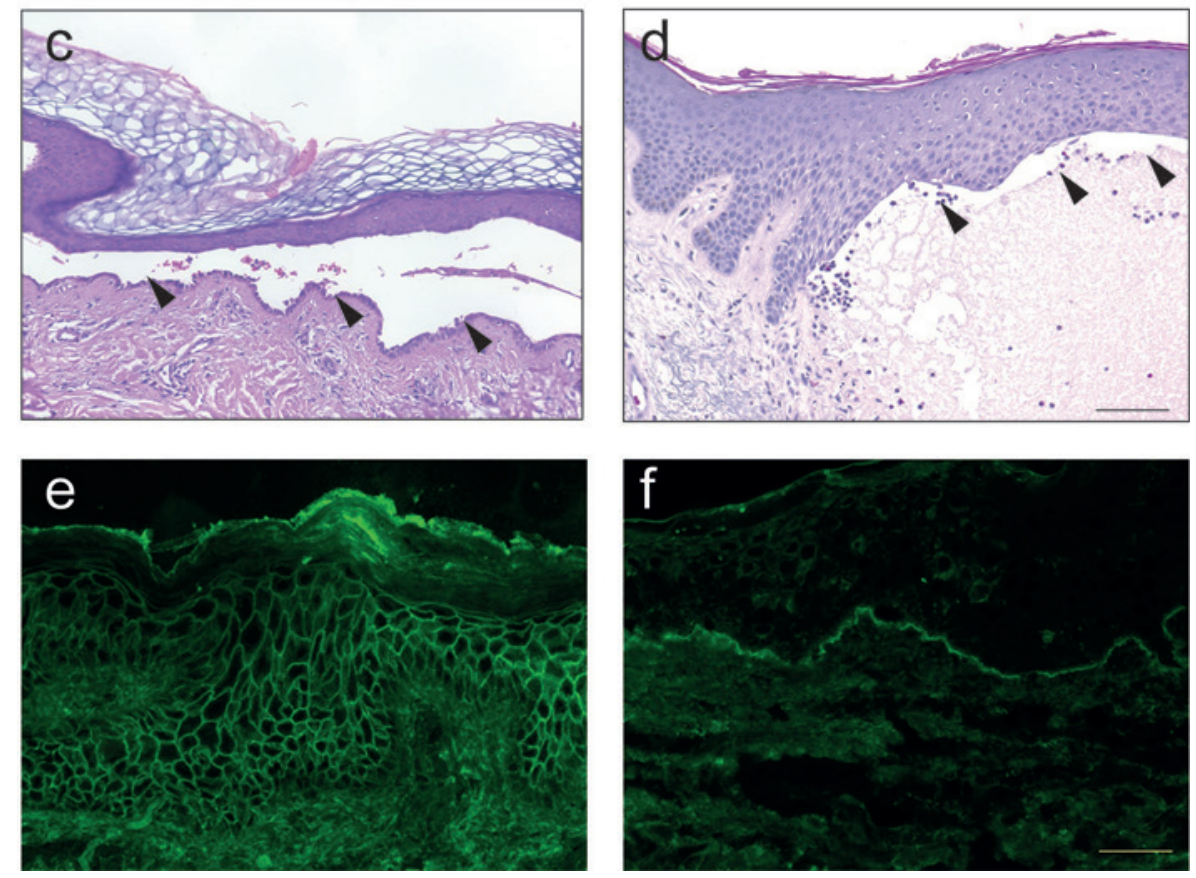

1 Identifizierung unterschiedlicher Muster von Autoantikörperablagerungen in der Haut

2 Entdeckung spezifischer Autoantigene bei verschiedenen Pemphigus- und Pemphigoid-Erkrankungen

3 Definition von Pemphigus und Pemphigoid als Autoimmunkrankheiten

4 Etablierung der derzeitigen molekularen Diagnose von Pemphigus und Pemphigoid

5 Erforschung der funktionell relevanten Moleküle und Signalwege mithilfe komplexer Modellsysteme

6 Definition eindeutiger Pemphigus- und Pemphigoidvarianten entsprechend der Epidemiologie

7 Entwicklung in Richtung einer personalisierten Behandlung, die selektiv spezifische, autoreaktive B-Zellen angreift

Diese Meilensteine der personalisierten Medizin bei Pemphigus und Pemphigoid werden in den folgenden Abschnitten ausführlich erläutert.

\section{Histologische Differenzierung zwischen Pemphigus und Pemphigoid}

Seit Hippokrates (460-370 v. Chr.), der verschiedene Arten von Fieber in Verbindung mit Blasenbildung als «Pemphigusfieber» beschrieb, wurde der Begriff «Pemphigus» als deskriptive Bezeichnung für Hautkrankheiten verwendet, die durch Blasenbildung gekennzeichnet sind. In seiner heutigen Bedeutung wurde der Begriff Pemphigus allerdings 1791 von Dr. Wichman geprägt, der einen Fall von Pemphigus (gemäß dem heutigen Verständnis) beschrieb. In der Folgezeit wurde «Pemphigus» als gleichbedeutende Bezeichnung für sämtliche vesikulären oder bullösen Erkrankungen verwendet. Dies führte zur Entstehung mehrerer unterschiedlicher Arten von «Pemphigus» [14]. Das von Wichman aufgestellte Konzept wurde im Jahr 1860 von Dr. Hebra bekräftigt, der feststellte, dass es sich bei Pemphigus stets um eine chronische Erkrankung handelt. Mithin wurden alle Pemphigus- und Pemphigoid-Erkrankungen unter dem Begriff «Pemphigus» zusammengefasst [15]. Entsprechend der Prognose wurden zwei verschiedene Formen von «Pemphigus» unterschieden: maligner und benigner Pemphigus. In seinen 1953 veröffentlichten wegweisenden histologischen Beobachtungen unterschied Walter Lever zwischen Pemphigus, der durch eine intraepidermale Blasenbildung gekennzeichnet ist, und BP, bei dem es zu einer subepidermalen Blasenbildung kommt [13] (Abbildung 2). Er bezog zudem das klinische Bild mit ein und prägte den Begriff «Schleimhautpemphigoid» (mucous membrane pemphigoid, MMP), das durch eine ähnliche Histologie wie BP gekennzeichnet ist, jedoch mit Blasen- 
Tab. 1. Autoantigene bei Pemphigus und Pemphigoid

\begin{tabular}{|c|c|c|c|}
\hline & Erkrankung & Hauptzielantigen(e) & Hauptisotyp(en) \\
\hline \multirow[t]{5}{*}{ Pemphigus } & $\begin{array}{l}\text { Pemphigus vulgaris (PV) } \\
\text { Pemphigus foliaceus (PF) }\end{array}$ & $\begin{array}{l}\text { Dsg } 3 \text { bei mukosalem PV, Dsg } 1 \text { und } 3 \text { bei mukokutanem PV } \\
\text { Dsg } 1\end{array}$ & $\begin{array}{l}\lg G \\
\lg G\end{array}$ \\
\hline & Paraneoplastischer Pemphigus (PNP) & Envoplakin, Periplacin, Dsg 1/3, BP180 u. a. & $\lg G$ \\
\hline & IgA-Pemphigus & Dsg 1/3, Dsc 1-3 & $\lg A$ \\
\hline & Endemischer Pemphigus foliaceus & Dsg 1 & $\lg G$ \\
\hline & Herpetiformer Pemphigus & Dsg 1 & $\lg G$ \\
\hline \multirow[t]{6}{*}{ Pemphigoid } & $\begin{array}{l}\text { Bullöses Pemphigoid (BP) } \\
\text { Schleimhautpemphigoid (MMP) }\end{array}$ & $\begin{array}{l}\text { BP180-NC16A, BP230 } \\
\text { BP180, BP230, Laminin-332, a4ß6-Integrin, Laminim-331, COL7 }\end{array}$ & $\begin{array}{l}\lg G / \lg E \\
\lg G\end{array}$ \\
\hline & Pemphigoid gestationis (PG) & BP180-NC16A & $\lg G$ \\
\hline & Lineare IgA-Dermatose (LAD) & LAD-1 & $\lg A$ \\
\hline & Epidermolysis bullosa acquisita (EBA) & COL7 & $\operatorname{lgG} / \lg A$ \\
\hline & Anti-p200-Pemphigoid & Laminin $\gamma 1$ (nicht-pathogene Autoreaktivität) & $\lg G$ \\
\hline & Lichen planus pemphigoides & BP180-NC16A, BP230 & $\lg G$ \\
\hline
\end{tabular}

Hinweis: Pemphigus kann auch durch Nicht-Dsg-Autoantikörper induziert werden [33, 34], doch weisen mehr als 95 \% der Patienten mit Pemphigus vulgaris/ foliaceus Anti-Dsg-Autoantikörper auf [35, 36]. Daher wurden die Nicht-Dsg-Autoantikörper nicht in diese Tabelle aufgenommen. Dsg: Desmoglein; Dsc: Desmocollin; COL7: Typ VII-Kollagen; LAD-1: lineare IgA-Dermatose Antigen-1 (lösliche Ektodomäne von BP180); MMP: mucous membrane pemphigoid (Schleimhautpemphigoid).

bildung an den Schleimhäuten einhergeht [13]. Walter Levers genaue klinische und histologische Beobachtungen sind nach wie vor gültig und ein gutes Beispiel dafür, wie sorgfältige klinische Beobachtungen wegweisende Entdeckungen ermöglichen.

\section{Identifizierung spezifischer Muster von Autoantikörper- ablagerungen bei Pemphigus und Pemphigoid}

Etwa zwanzig Jahre nach der Entdeckung von Dr. Lever wurden bei Patienten mit Pemphigus [16] und Pemphigoid [17] IgG-Ablagerungen nachgewiesen. Bei Pemphigus fand man Immunglobulin (Ig)-Ablagerungen an der Oberfläche der Keratinozyten. In der Folge wurde für dieses spezielle Färbemuster daher die Bezeichnung «honigwabenartige» Fluoreszenz innerhalb der Epidermis geprägt (Abbildung 2). Bei Pemphigoid-Erkrankungen finden sich lineare Ig/Komplement (C)-Ablagerungen entlang der dermoepidermalen Junktionszone. Dieses Färbemuster wird als «lineare» Ig/C-Ablagerung bezeichnet (Abbildung 2). Kürzlich wurden bei Pemphigoid-Erkrankungen spezifische Muster von IgG-Ablagerungen nachgewiesen. Im Gegensatz zu allen anderen Pemphigoid-Erkrankungen, bei denen die IgG-Ablagerungen ein «n-gezacktes» Verteilungsmuster zeigen, findet sich bei der Pemphigoid-Erkrankung EBA ein «u-gezacktes» Muster von IgG und IgA [18-20]. Die Identifizierung dieser Muster in der direkten Immunfluoreszenzmikroskopie (IF) ist eine erlernbare Fertigkeit [21] und für die Diagnose von EBA entscheidend, da bis zu $60 \%$ der Patienten seronegativ sind [22]. Der Nachweis dieser spezifischen Färbemuster bei Pemphigus und Pemphigoid (sowie bei EBA) stützten die 1953 gemachte histopathologische Beobachtung, nach der es sich bei Pemphigus und Pemphigoid um unterschiedliche Krankheiten handelt.

\section{Definition von Pemphigus und Pemphigoid als Autoim- munkrankheiten}

Die Ablagerung von IgG in der Haut legte die Vermutung nahe, dass Pemphigus und Pemphigoid möglicherweise durch eine Immunreaktion auf Autoantigene hervorgerufen werden. Es fehlte jedoch ein direkter Beleg für die Autoimmunpathogenese von Pemphigus und Pemphigoid. Ein solcher direkter Beweis ist gemäß den revidierten Witebsky-Postulaten die Auslösung der Krankheit bei Versuchstieren durch Übertragung von Patientenserum oder -IgG [23]. Der Autoimmuncharakter des Pemphigus wurde 1982 von Grant Anhalt und Kollegen nachgewiesen, die durch den Transfer von IgG von Pemphigus-Patienten in neonatale Balb/c-Mäuse eine Blasenbildung und Erosionen der Haut auslösten, die mit histologischen, ultrastrukturellen und immunologischen Befunden verbunden waren, wie sie bei PemphigusPatienten zu finden sind [24]. Versuche, die Pemphigoid-Krankheit BP durch Transfer von Patienten-IgG in Mäuse zu reproduzieren, blieben erfolglos, da es nicht zu einer Bindung des übertragenen IgG an die Maus-Haut kam [25]. Mehrere Jahre später, im Jahr 1993, nachdem die Autoantigene bei BP beschrieben worden waren (BP180 und BP230) und die fehlende Kreuzreaktivität menschlicher Autoantikörper mit dem murinen BP180 nachgewiesen worden war, gelang es Zhiou Liu und Kollegen, durch den Transfer von Kaninchen-Anti-Maus-BP180-IgG in Mäuse ein experimentelles BP zu induzieren [26]. Die Definition von Pemphigus und (später) Pemphigoid als Autoimmunerkrankungen und der Nachweis unterschiedlicher klinischer, histologischer und immunologischer Merkmale bei experimentellem Pemphigus und Pemphigoid waren der Auslöser für die Suche nach Autoantigenen. In der Folge wurde die pathogene Bedeu- 
tung verschiedener Autoantigene und ihrer entsprechenden Antikörper in verschiedenen Mausmodellen für Pemphigus foliaceus (PF), paraneopalastischen Pemphigus (PNP), MMP und EBA nachgewiesen.

\section{Identifizierung unterschiedlicher Autoantigene bei Pem- phigus und Pemphigoid}

Die Identifizierung von Ig-Ablagerungen in der Haut sowie der Nachweis der Autoimmunpathogenese von Pemphigus und Pemphigoid gaben den Anstoß für die Suche nach mutmaßlichen Autoantigenen, die weiterhin andauert, wie etwa im Fall des Anti-p200-Pemphigoids [27]. Bereits 1988 wurde COL7 mit Hilfe von Autoantikörpern von Patienten und monoklonalen Antikörpern gegen Typ VII-Kollagen (COL7) als das Autoantigen bei EBA identifiziert [28]. Im Jahr 1990 erfolgte die Klonung von BP180 beim Menschen aus einer Keratinozytenbibliothek [29]. Ein Jahr später wurden Desmoglein (Dsg) 1 und 3 geklont und als Autoantigene bei PF und Pemphigus vulgaris (PV) identifiziert [3032]. In der Folge wurden weitere Autoantigene bei Pemphigus und Pemphigoid geklont und/oder identifiziert (Tabelle 1).

\section{Moderne molekulare Diagnose von Pemphigus und Pem- phigoid}

Der Nachweis unterschiedlicher Autoantigene bei Pemphigusund Pemphigoid-Erkrankungen ermöglichte die Entwicklung der derzeit eingesetzten molekularen Diagnostik bei Pemphigus und Pemphigoid [37]. Goldstandard für die Diagnose von Pemphigus und Pemphigoid bei entsprechendem klinischen Verdacht ist der Nachweis von gewebegebundenen Autoantikörpern (oder C3) in einer periläsionalen Haut- (oder Schleimhaut-)Biopsie. Je nach Lokalisation der Ig- oder C3-Ablagerungen unterscheidet man zwischen Pemphigus (Ablagerungen im Interzellularraum, so genanntes Honigwabenmuster) und Pemphigoid (lineare Färbung entlang der dermoepidermalen Junktionszone). Eine weitere Differenzierung der EBA kann anhand des Zackenmusters vorgenommen werden [19]. Die Identifizierung dieser Muster ist eine erlernbare Fertigkeit und von entscheidender Bedeutung, da die EBA in bis zu 60\% der Fälle seronegativ ausfallen kann [21, 22]. Wenn es nicht möglich ist, die Diagnose mittels direkter IF-Mikroskopie zu stellen, kann eine indirekte IF-Mikroskopie unter Verwendung verschiedener Organsubstrate (meist Affenösophagus und mit Salzlösung separierte menschliche Spalthaut) durchgeführt werden, um weiter zwischen den verschiedenen Pemphigus- und Pemphigoid-Erkrankungen zu differenzieren [37]. Andere, weniger häufig verwendete Substrate sind Rattenblase bei PNP und normale Mundschleimhaut bei MMP [38]. Während für den Nachweis zirkulierender Pemphigus-bezogener Autoantikörper Affenösophagus hilfreich ist, binden Pemphigoid-Autoantikörper besser an Salzspalthaut. Hervorzuheben ist, dass bei einer linearen Ablagerung von Autoantikörpern des Patienten am Blasendach der artefiziell gespaltenen Haut ein BP (oder MMP bei Patienten mit überwiegender Schleimhautbeteiligung) diagnosti- ziert wird, da die entsprechenden Autoantigene (BP180 und BP230) im Blasendach exprimiert werden. Laminin-332, COL7 und p200 werden dagegen im Blasengrund exprimiert. Daher ist, wenn sich in der indirekten IF-Mikroskopie auf Salzspalthaut eine Bindung von Autoantikörpern des Patienten im Blasengrund findet, eine weitere Differenzierung zwischen MMP-, EBA- und Anti-p200-Pemphigoid erforderlich. Letztere und die (semiquantitative) Bestimmung der Konzentration an zirkulierenden Autoantikörpern kann durch den Nachweis spezifischer Autoantikörper erreicht werden. Hierzu können kommerzielle ELISA-Systeme oder Biochip-Mosaike unter Verwendung der rekombinanten immundominanten Domänen der Zielantigene, d. h. Dsg1, Dsg3, Envoplakin, BP180, BP230, Laminin 332 und COL7 eingesetzt werden. Darüber hinaus haben spezialisierte Labors Verfahren (vor allem Wester-Blotting oder Immunpräzipitation) zum Nachweis von Autoantikörpern gegen p200, ausgewählte Ketten von Laminin-332, die Ektodomäne von BP180 oder seltene Autoantigene entwickelt. Mithilfe dieser modernen molekularen Diagnostik von Pemphigus und Pemphigoid lassen sich (in den meisten Fällen) die jeweils vorliegenden Pemphigus- und Pemphigoid-Erkrankungen diagnostizieren. Vor dem Hintergrund, dass große Unterschiede hinsichtlich der Behandlung und Prognose bestehen $[2,3]$ ist es dadurch möglich, die adäquate Behandlung für den einzelnen Patienten zu auswählen. Neben der Anwendung in der Diagnostik gestattet die Langzeitkontrolle eine Früherkennung von Rezidiven, da die Konzentration der zirkulierenden Autoantikörper intraindividuell mit der Krankheitsaktivität korreliert [39-41]. Die molekulare Charakterisierung einiger AIBD ist jedoch weiterhin nur in hochspezialisierten wissenschaftlichen Zentren möglich. Dadurch erhalten die Patienten unter Umständen erst mit Verzögerung die richtige Diagnose und die optimale Behandlung.

Eine aktuelle Entwicklung in der personalisierten Behandlung von Pemphigoid ist die Identifizierung von anderen Biomarkern als den Autoantikörpern, mit deren Hilfe es möglich ist, das Ansprechen auf die Behandlung und/oder ein Rezidiv vorherzusagen [42]. Kurz gesagt war die Persistenz erhöhter ECP (eosinophiles kationisches Protein)-Werte bei Patienten mit BP mit einem Rezidiv assoziiert [43]. Neben anhaltend hohen ECP-Konzentrationen sind auch das Vorliegen von Anti-COL7-Autoantikörpern (der Autoantikörper bei EBA) (44) und erhöhte CXCL10-Serumspiegel [45] prädiktive Faktoren für ein Rezidiv bei Patienten mit BP. Gegenüber nicht-autoreaktiven B-Zellen zeigte sich bei autoreaktiven B-Zellen von Patienten mit PV eine Überexpression von Genen, die für die pro-inflammatorischen Zytokine IL-1 $\beta$, IL23p19 und IL-12p35 sowie den Transkriptionsfaktor IRF5 kodieren. Im Vergleich zu Patienten mit aktivem Pemphigus war bei Patienten, die nach Gabe von Rituximab eine Vollremission erreichten, eine Unterexpression von IL-1 $\beta$ und den CD27-Gedächtniszellmarker-Genen zu beobachten [46].

Es wurden umfangreiche Anstrengungen unternommen, um einen personalisierten Ansatz zur Optimierung der Behandlung von Pemphigus-Patienten zu etablieren, der es ermöglicht vorherzusagen, welche Patienten mit einer Prädisposition für ein frühes 
Rezidiv unter Rituximab von einer Rituximab-Dosis als Erhaltungstherapie in Monat 6 profitieren. Es zeigte sich, dass ein erhöhter Schweregrad bei Baseline und erhöhte Anti-Dsg1- und Anti-Dsg3-Werte drei Monate nach der ersten Infusion unabhängige Prädiktoren für ein frühes Rezidiv nach Behandlung mit Rituximab waren, weshalb eine Rituximab-Erhaltungsdosis nach sechs Monaten in Betracht gezogen werden sollte [47].

\section{Funktionell relevante Moleküle und Signalwege bei Pem- phigus und Pemphigoid}

Mithilfe von Keratinozytenkulturen, ex-vivo-Hautmodellen und den oben beschriebenen Mausmodellen [48, 49] wurden verschiedene Krankheitsmechanismen identifiziert, die die Grundlage für wertvolle neuartige Therapieansätze bilden. Desmogleine (Dsgs) sind transmembrane desmosomale Cadherin-ähnliche Glykoproteine, die die Gewebeintegrität aufrechthalten und die Zell-Zell-Adhäsion erleichtern. Die wichtigste ätiopathogenetische Rolle bei der Entstehung von PV bzw. PF spielen IgG-Autoantikörper, die gegen Dsg3 und Dsg1 gerichtet sind. Bei Pemphigus reichen monovalente Fragmente von Anti-Dsg-Antikörpern, denen der Fc-Teil fehlt, aus, um in vitro und in vivo eine Akantholyse zu verursachen [50]. Die genaue Abfolge der Ereignisse bei der anti-Dsg-Antikörper-vermittelten Akantholyse ist bislang nicht vollständig geklärt. Drei Hauptereignisse nach der Bindung von Anti-Dsg-IgG wurden beschrieben: (I) direkte Interferenz mit der Dsg-Transinteraktion, ein Phänomen, das als sterische Hinderung bezeichnet wird, (II) Remodeling der Dsg-Expression auf der Zelloberfläche, das zu einer Internalisierung und Depletion von Dsg von der Zellmembran führt und (III) Signalereignisse, die sich störend auf die Architektur des Zytoskeletts auswirken $[3,4]$. Diese Mechanismen gelten nicht in gleicher Weise für die Bindung von Dsg1 und Dsg3 an IgG. Die Bindung von Dsg1 führt zu einem Einstrom von $\mathrm{Ca} 2+$ und zu einer Aktivierung des ERKSignalwegs. Demgegenüber führt die Bindung von Dsg3-spezifischem IgG zu einer Signalübertragung über p38MAPK in der Epidermis, nicht jedoch in den Schleimhäuten, und die Proteintyrosinkinasen der SRC-Familie sowie die EGFR-Signalwege werden aktiviert [51, 52]. Überdies deuten aktuelle Daten stark darauf hin, dass neben den Dsg1/3-Autoantikörpern auch Nicht-DsgAntikörper sowie der lösliche Fas-Ligand beim Pemphigus-Phänotyp eine Rolle spielen [53-56].

Anders als bei Pemphigus-Erkrankungen sind bei PemphigoidErkrankungen Fc $\gamma$ R-vermittelte Effekte entscheidend für die Blasenbildung, und in den letzten zehn Jahren wurden verschiedene Krankheitswege und Schlüsselmoleküle beschrieben, die bei diesen Erkrankungen funktionell relevant sind, darunter mehrere Signalmoleküle, Leukotrien B4 (LTB4) und IL-17 [57-60]. Zudem ist allgemein anerkannt, dass die Komplementaktivierung an der dermepidermalen Junktionszone eine tragende Säule bei der Rekrutierung von Neutrophilen, Eosinophilen und Makrophagen an dieser Stelle ist [61]. Hervorzuheben ist, dass hinsichtlich der Auswirkungen der Komplementaktivierung leichte Unterschiede zwischen den verschiedenen Pemphigoid-Erkrankungen sowie zwischen den klinischen BP-Varianten bestanden. Dagegen scheint die Akantholyse bei Pemphigus unabhängig von der Komplementaktivierung aufzutreten, obgleich die Anfärbung von C3 im Epithel/in der Epidermis ein diagnostisches Kennzeichen ist [3]. Durch die Erforschung dieser Unterschiede lassen sich möglicherweise Untergruppen von Patienten und Krankheiten aufdecken, bei denen therapeutische Interventionen, die auf die Komplementkomponenten abzielen, von Nutzen sein können. Bei etwa $80 \%$ der BP-Patienten finden sich C3c-Ablagerungen entlang der dermoepidermalen Junktionszone in periläsionalen Biopsien. Patienten mit C3c-Ablagerungen wiesen signifikant höhere Anti-BP180-NC16A-IgG-Serumspiegel auf, und Patienten ohne Blasen hatten signifikant weniger C3c-Ablagerungen entlang der dermoepidermalen Junktionszone [62]. Es wurde kein Zusammenhang zwischen dem Ausmaß der Hautläsionen und der C3c-Färbung in der Haut der Patienten festgestellt, wohingegen die Komplementaktivierungskapazität der Autoantikörper im ex-vivo-Komplementbindungsassay mit der Krankheitsaktivität korrelierte, die mit dem Bullous Pemphigoid Disease Area Index (BPDAI) ermittelt wurde [63]. Der Nachweis erhöhter C3aSpiegel im Serum von BP-Patienten und die positive Korrelation der C3a-Serumkonzentrationen mit Anti-BP180-NC16A-IgG und löslichem CD46, einem komplementregulatorischen Protein mit zentraler Bedeutung bei der Komplementaktivierung, liefern weitere Belege für die Bedeutung der Komplementaktivierung bei BP [64]. Allerdings bestehen keine Unterschiede in den Plasmakonzentrationen von C3a, C4a und C5a zwischen BP-Patienten und nach Alter und Geschlecht vergleichbaren Kontrollen. Überdies bleiben die Plasmaspiegel dieser drei Komplementkomponenten bei Messung während eines BP-Schubs und einer Remission des BP konstant [65]. Im neonatalen BP-Mausmodell, bei dem die Blasen typischerweise 24 bis 48 Stunden nach der Injektion von Kaninchen-IgG gegen die NC15A-Domäne von BP180 auftreten, war der Phänotyp der Blasenbildung vollständig von der Komplementaktivierung an der dermoepidermalen Junktionszone abhängig $[66,67]$. Die gleiche Komplementabhängigkeit zeigte sich in einem humanisierten Mausmodell des BP, in dem die homologe murine NC15A-Region durch die humane NC16ADomäne ersetzt worden war [68]. In einem anderen humanisierten Mausmodell des BP, in dem das vollständige BP180-Molekül durch das menschliche Protein ersetzt worden war, führte die Injektion von polyklonalem F(ab')2 Anti-NC16A-IgG oder nicht komplementaktivierendem Anti-NC16A-IgG4 dagegen zu einer Blasenbildung $[69,70]$. Übereinstimmend damit berichteten Dainichi et al. über zwei BP-Patienten ohne C3-Ablagerungen in der Haut und IgG4-Autoantikörper als Hauptunterklasse, die ex vivo nicht in der Lage waren, eine Komplementaktivierung auszulösen [71]. Weitere Beobachtungen in diesem Modell mit C3-defizienten Tieren, die für die pathogene Wirkung von Anti-BP180-IgG empfänglich waren, und aktuell in einem BP-Modell mit adulten Mäusen, bei dem der Transfer von Anti-BP180-NC15A-IgG in C5aR1-defiziente Mäuse einen 50\%igen Rückgang der Hautläsionen bewirkte [70, 72, 73], sprechen für komplementunabhängige Mechanismen in der Pathophysiologie des BP. Interessanterweise
160

Kompass Autoimmun 2021;3:155-162 DOI: $10.1159 / 000519459$ 
schien die Komplementaktivierung im adulten Mausmodell von EBA und Anti-Laminin-332-MMP, ähnlich wie beim neonatalen BP-Mausmodell, eine Voraussetzung für den blasenbildenden Phänotyp zu sein [74-76]. Die Erforschung des komplexen Szenarios der Komplementaktivierung bei Pemphigoid-Erkrankungen wird zweifellos dazu beitragen, Untergruppen von Patienten zu identifizieren und spezifischere sichere maßgeschneiderte Behandlungen für diese Krankheiten zu entwickeln. Die dosisabhängige Hemmung der bei BP auftretenden IgG-induzierten C3Ablagerung an der dermoepidermalen Junktionszone bei der invitro-Komplementbindung an Kryoschnitten menschlicher Haut durch (I) den Anti-C1s-Antikörper TNT003, (II) das niedermolekulare Heparin Tinzaparin-Natrium und (III) den dualen C5/ LTB4-Antagonisten Coversin, die alle die normale Aktivität des Komplementwegs stören $[65,77,78]$, ist für uns ein Grund, die Komplementhemmung als hilfreichen therapeutischen Angriffspunkt bei BP näher zu untersuchen.

\section{Die Epidemiologie definiert spezielle Formen innerhalb der verschiedenen Pemphigus- und Pemphigoid-Erkran- kungen}

Wie der Name vermuten lässt, ist der PV der Pemphigus-Subtyp mit der höchsten Prävalenz und macht etwa $70 \%$ aller Fälle aus [79]. Der PV ist durch eine unterschiedliche geografische und ethnische Verteilung gekennzeichnet und die jährlichen Inzidenzraten in verschiedenen Regionen betragen zwischen 0,8 und 16,1 Fällen pro eine Million Einwohner [80]. In Übereinstimmung damit weisen einige ethnische Gruppen, speziell aschkenasische Juden und Menschen aus dem Mittelmeerraum, Berichten zufolge eine Prädisposition für die Entwicklung von Pemphigus auf [80]. Eine aktuelle populationsbasierte Studie in Israel zeigte, dass die Inzidenz von PV bei Juden gegenüber Arabern um das 3,6Fache erhöht war [81]. In einer im US-Bundesstaat Connecticut durchgeführten retrospektiven Studie fiel die jährliche Inzidenz von PV bei Menschen jüdischer Abstammung fast achtmal höher aus als bei Angehörigen anderer ethnischer Gruppen [82]. Diese epidemiologischen Beobachtungen wurden durch genetische Untersuchungen gestützt, die eine Assoziation zwischen verschiedenen HLA-Klasse-II-Genen, HLA-DRB1 ${ }^{\star} 04$ und HLA-A ${ }^{\star} 10$, und dem Auftreten von PV bei aschkenasischen Juden nachwiesen [83-85]. In der Folgezeit wurde eine polymorphe Variante im ST18-Gen bei jüdischen und ägyptischen, nicht jedoch bei deutschen Patienten mit PV in Verbindung gebracht [86]. Daher besteht zwar eine zugrunde liegende genetische Prädisposition für PV, doch kann sich die Krankheit auch manifestieren, ohne dass bestimmte genetische prädisponierende Faktoren vorliegen. Dies alles deutet auf ein polygenes genetisches Risiko für die Entwicklung von PV hin und spricht dafür, dass die Umwelt ein potenzieller treibender Faktor bei der Pathogenese der Krankheit ist, wie dies auch für andere entzündliche Erkrankungen berichtet wurde [87].

Der sporadische PF ist eine seltene Erkrankung, die 20 bis 30\% aller Pemphigus-Fälle ausmacht. Schätzungen zufolge beträgt die jährliche Inzidenz in Europa und den Vereinigten Staaten weniger als ein Fall pro eine Million Einwohner [80]. In brasilianischen, italienischen, französischen und niederländischen Populationen war HLA-DRB1 ${ }^{\star} 4$ mit einem erhöhten Risiko für sporadischen $\mathrm{PF}$ assoziiert [88, 89]. Bei Patienten mexikanischer Herkunft mit sporadischem PF wurde ein Zusammenhang mit HLA-DRB1 ${ }^{\star} 0101$ festgestellt [90]. In Israel war dagegen keine ethnische Prädilektion für das Auftreten von PF zu beobachten, da die bereinigte Inzidenzrate der Krankheit bei Juden und Arabern vergleichbar ausfiel [81]. Neben sporadischen Fällen wurden endemische PF-Subtypen in Brasilien (fogo selvagem), Kolumbien und Tunesien beschrieben [80]. Klinisch, histologisch und immunologisch sind Patienten mit endemischem PF nicht von denjenigen mit sporadischem PF zu unterscheiden [80, 91], allerdings ist ersterer durch ein geografisches Verteilungsmuster, eine familiäre Prädisposition und ein jüngeres Erkrankungsalter gekennzeichnet $[92,93]$.

Die weltweit häufigste subepidermale AIBD ist das BP, das typischerweise bei älteren Menschen, vor allem bei über 75-Jährigen, auftritt. Während die allgemeine jährliche Inzidenz von BP Berichten zufolge zwischen 2,4 und 23,0 Fällen pro eine Million Einwohner liegt, nimmt sie bei den über 80-Järigen exponentiell auf 312 Fälle pro eine Million Einwohner zu [94]. Es liegen zunehmend Hinweise vor, nach denen die Inzidenz von BP in den letzten zwanzig Jahren deutlich um das 1,9- bis 4,3-Fache gestiegen ist [95]. Für diese Beobachtung wurden verschiedene mögliche Erklärungen postuliert, von denen eine zunehmende Exposition gegenüber bestimmten Medikamenten, die an der Induktion des $\mathrm{BP}$ beteiligt sind, am plausibelsten ist [95]. Als ein möglicher BPAuslöser wurden Dipeptidyl-Peptidase-IV-Ihibitoren (DPP4I), die so genannten Gliptine, eine kürzlich eingeführte Klasse oraler Antidiabetika, identifiziert [96-98]. Die abschließende Klärung der Frage, ob Patienten mit DPP4I-assoziiertem BP ein charakteristisches klinisches und immunologisches Profil aufweisen, steht noch aus. Während in europäischen Studien bei diesen Patienten keine speziellen Merkmale gefunden wurden, die sie von typischen BP-Patienten unterschieden, wiesen japanische Patienten mit DPP4I-assoziiertem BP eher ein nicht-inflammatorisches BP auf und die immundominante Domäne des BP180-Antigens (NC16A) war bei ihnen weniger häufig das Angriffsziel [99-101]. Die sorgfältige klinische Beobachtung hat dazu geführt, dass ein eindeutiges klinisches Bild des BP identifiziert wurde. Sollten sich die initialen Beobachtungen eindeutiger immunologischer und genetischer Merkmale des DPP4I-assoziierten BP bestätigen, würde dies ermöglichen, die Patienten entsprechend der zugrunde liegenden krankheitsfördernden Mechanismen zu behandeln. Bei DPP4I-assoziiertem BP ist dies relativ einfach, da das BP normalerweise nach Absetzen der DPP4I-Behandlung abklingt.

\section{CAAR-T-Zellen als potenzielles und personalisiertes Heil- mittel bei Pemphigus und Pemphigoid}

Der neueste Fortschritt in Richtung eines personalisierten Therapieansatzes ist die Entwicklung chimärer Autoantigenrezeptor 
(CAAR)-T-Zellen zur Behandlung von Pemphigus [102]. Ausgehend von der bahnbrechenden Entdeckung chimärer Antigenrezeptor (CAR)-T-Zellen zur Behandlung maligner hämatologischer Erkrankungen [103] entwickelten Aimee Payne und Kollegen einen rekombinanten T-Zell-Rezeptor, indem sie das bei PV vorliegende Autoantigen (Dsg3) mit CD137-CD3 $\zeta$ fusionierten. Diese Dsg3-CAAR-T-Zellen verfügten über eine spezifische Zytotoxizität gegen B-/Plasmazellen, die den für Dsg3 spezifischen B-Zell-Rezeptor exprimieren. Anschließend wurde durch Transfer von Dsg3-Hybridom-Zelllinien in Mäuse bei diesen ein experimenteller Pemphigus induziert. Wenn die Mäuse zusätzlich Dsg3-CAAR-T-Zellen injiziert bekamen, waren sie vor der Induktion eines experimentellen Pemphigus geschützt [102]. Nachfolgend dehnte die Gruppe ihre Untersuchungen auf weitere Autoantigene bei Pemphigus, speziell Dsg1, aus [104]. Gegenwärtig läuft eine klinische Phase-I-Studie mit autologen Dsg3-CAAR-TZellen bei mukosalem PV (https://cabalettabio.com/clinical-trials/, Zugriff am 31. Mai 2020). Mithilfe der selektiv auf autoreaktive B-Zellen ausgerichteten CARR-T-Zelltechnologie wird derzeit ein hochgradig personalisierter Behandlungsansatz für PV entwickelt [105]. Sollten diese (und weitere) klinische Studien erfolgreich abgeschlossen werden, wird dies eine neue Ära in der Behandlung von B-Zell-assoziierten Autoimmunerkrankungen [106] einläuten.

\section{Zukunftsperspektiven}

Es ist davon auszugehen, dass die Entwicklung von Therapien, die beispielsweise mithilfe von CAAR-T-Zellen selektiv autoreaktive $\mathrm{B}-Z$ Zellen angreifen, die Behandlung des Pemphigus enorm verändern wird. Besonders interessant ist die Einschätzung, ob CAART-Zellen bei Pemphigoid-Erkrankungen das gleiche Potenzial haben. Alternativ könnte die Immunisierung von PV-Patienten mit Dsg3-beschichteten Nanopartikeln die Autoimmunität gegen Dsg3 spezifisch unterdrücken und dieser Ansatz wird derzeit in einer klinischen Phase-I-Studie bei PV mit Dsg3 verfolgt. Die Verwendung von IL-17 und Eotaxin als Zielstrukturen wird derzeit als ein potenzieller therapeutischer Ansatz bei BP untersucht [107]. Der Erfolg dieser Therapien wird jedoch von einer Auswei- tung der molekularen Diagnostik abhängen, durch die es möglich wird, die Autoimmunreaktion beim einzelnen Patienten genau zu bestimmen. Parallel dazu ist zu erwarten, dass die molekulare Diagnostik helfen wird, verschiedene (Unter-)Gruppen von Pemphigus- und Pemphigoid-Erkrankungen zu definieren, die höchstwahrscheinlich immer stärker auf molekularen Signaturen basieren werden. Langfristig betrachtet ist es vorstellbar, dass kurative und sichere Therapien für Pemphigus und Pemphigoid zur Verfügung stehen werden.

\section{Beiträge der einzelnen Autoren}

K. Bi., K. K., S. E., K. Bo., E. S. und R. L. verfassten das Manuskript. Alle Autoren waren an dem Artikel beteiligt und haben die eingereichte Fassung genehmigt.

\section{Finanzierung}

Exzellenzcluster «Präzisionsmedizin für chronische Entzündungserkrankungen» (EXC 2167) der Deutschen Forschungsgemeinschaft.

\section{Disclosure Statement}

In den vergangenen 3 Jahren erhielt E. S. Forschungsgelder von Novartis, UCB, Incyte, Biotest, Euroimmun, Dompe, Admirx, Synthon, TxCell, ArgenX und Fresenius Medical Care sowie Beratungs- und Vortragshonorare und/oder Reisekostenerstattungen von Novartis, UCB, Biotest, TxCell, ArgenX, Roche, Genentech, Imevax, Amryth, Thermo Fisher, AstraZeneca, True North Therapeutics, Bristol-Myers Squibb, und Fresenius Medical Care. R. L. erhielt Forschungsgelder von Almirall, True North Therapeutics, UCB Pharma, ArgenX, TxCell, Topadur, Incyte und Admirx und Beratungs- oder Vortragshonorare von ArgenX, Immunogenetics, Novartis und Lilly.

Die übrigen Autoren erklären, dass bei der Durchführung der Forschungsarbeit keine kommerziellen oder finanziellen Beziehungen bestanden, die als potenzieller Interessenkonflikt ausgelegt werden könnten.

\section{Lizenzangabe}

Bieber K, Kridin K, Emtenani S, Boch K, Schmidt E, Ludwig RJ: Milestones in Personalized Medicine in Pemphigus and Pemphigoid. Front Immunol. 2021 Jan 11;11:591971 (DOI: 10.3389/fimmu.2020.591971). ${ }^{\circ} 2021$ Bieber, Kridin, Emtenani, Boch, Schmidt und Ludwig (Übersetzung), lizensiert unter CC BY 4.0 (https://creativecommons.org/licenses/by/4.0/deed.de).

\section{Literatur}

Die Literatur ist unter www.karger.com/doi/10.1159/000519459 abrufbar. 